\title{
Supplemental Information: \\ A Nanostructured non-Newtonian Drug Delivery Barrier Prevents Postoperative Intrapericardial Adhesions
}

Xichi Wang ${ }^{1,2,3,4} \S$, Zongtao Liu ${ }^{4} \S$, Diego A. Sandoval-Salaiza ${ }^{1,2,3,5}$, Samson Afewerki ${ }^{1,2,6}$, Mildred G. Jimenez-Rodriguez $1,2,3,5$, Lorena Sanchez-Melgar ${ }^{1,2,3,5}$, Gabriela GüemesAguilar $1,2,3,7$, David G. Gonzalez-Sanchez $z^{1,2,3,7}$, Oscar Noble ${ }^{1,2,3,7}$, Cecilia Lerma ${ }^{1,2,3,5}$, Roberto Parra-Saldivar ${ }^{1,2,5}$, Dario R. Lemos ${ }^{1,3}$, Guillermo A. Llamas-Esperon ${ }^{8}$, Jiawei $\mathrm{Shi}^{4}$, $\mathrm{Li} \mathrm{Li}^{3}$, Anderson O. Lobo ${ }^{9}$, Andres A. Fuentes-Baldemar ${ }^{1,2,3}$, Joseph $\mathrm{V}$. Bonventre ${ }^{1,2,3 *}$, Nianguo Dong ${ }^{4 *}$, Guillermo U. Ruiz-Esparza ${ }^{1,2,3 *}$

1 Division of Engineering in Medicine, Department of Medicine, Brigham and Women's Hospital, Harvard Medical School, Boston, MA, 02115, USA

2 Division of Health Sciences and Technology, Harvard University - Massachusetts Institute of Technology, Cambridge, MA, 02139, USA

3 Renal Division, Department of Medicine, Brigham and Women's Hospital, Harvard Medical School, Boston, MA, 02115, USA

4 Department of Cardiovascular Surgery, Union Hospital, Tongji Medical College, Huazhong University of Science and Technology, Wuhan, 430022, China

5 Tecnologico de Monterrey, School of Engineering and Science, Monterrey, NL, 64849, Mexico

6 Department of Chemical Engineering and Koch Institute for Integrative Cancer Research, Massachusetts Institute of Technology, Cambridge, MA, 02139, USA

7 Tecnologico de Monterrey, School of Medicine and Health Science, Monterrey, NL, 64849, Mexico

8 Department of Interventional Cardiology, Hospital Cardiológica, Aguascalientes, AGS, 20230, Mexico

9 LIMAV - Interdisciplinary Laboratory for Advanced Materials, Department of Materials Engineering, UFPI- Federal University of Piauí, Teresina, PI, 64049-550, Brazil

$\S$ These authors contributed equally to this work

${ }^{*}$ Corresponding Authors

\section{Address for Correspondence:}

Guillermo U. Ruiz-Esparza - Division of Health Sciences and Technology, Harvard University - Massachusetts Institute of Technology, Cambridge, MA, 02139, USA; Email: gruizeh@bwh.harvard.edu / gruizeh@mit.edu

Joseph V. Bonventre - Division of Engineering in Medicine, Department of Medicine, Brigham and Women's Hospital, Harvard Medical School, Boston, MA, 02115, USA; Email: joseph_bonventre@hms.harvard.edu

Nianguo Dong - Department of Cardiovascular Surgery, Un-ion Hospital, Tongji Medical College, Huazhong University of Science and Technology, Wuhan, 430022, China; Email: 1986xh0694@hust.edu.cn 


\section{Supplemental Material}

\section{Figure S1}
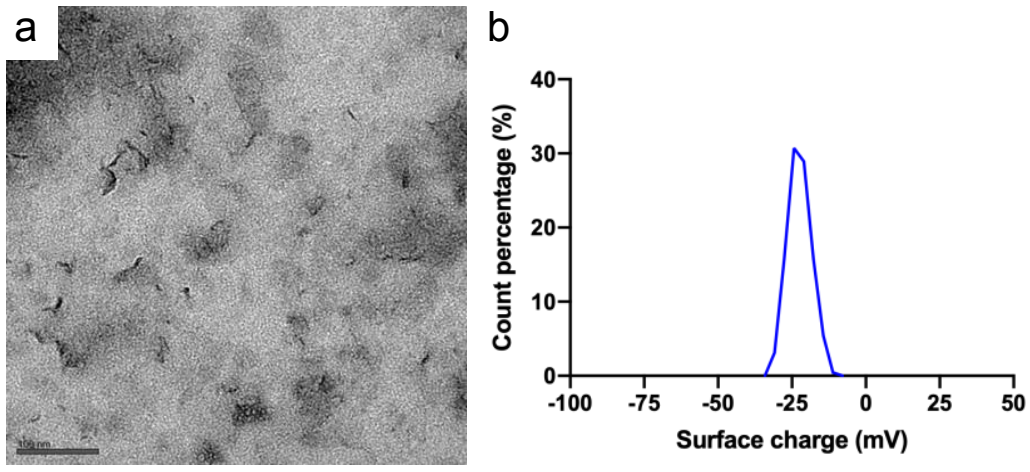

Fig. S1. Structural and physical characterization of SNDs. (a) Transmission electron microscopy (TEM) of SNDs showed elongated morphology with an average size of 25 $\mathrm{nm}$. Scale bar $=100 \mathrm{~nm}$. (b) Zeta potential analysis of SNDs was performed with a result of $-22.4 \mathrm{mV}$. 
Figure S2

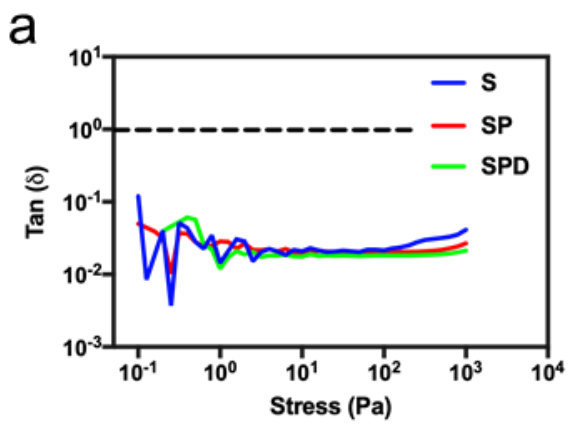

b

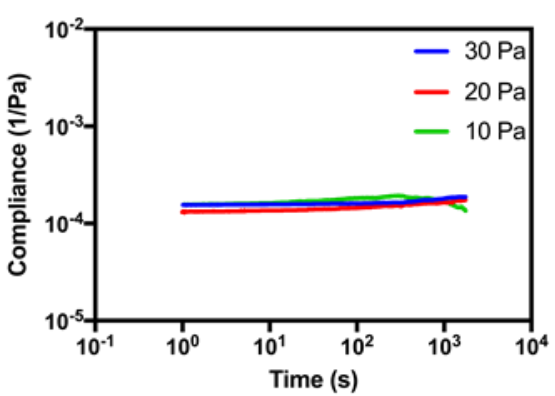

d

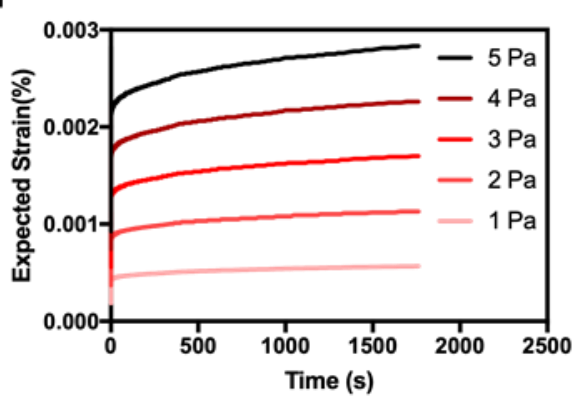

C

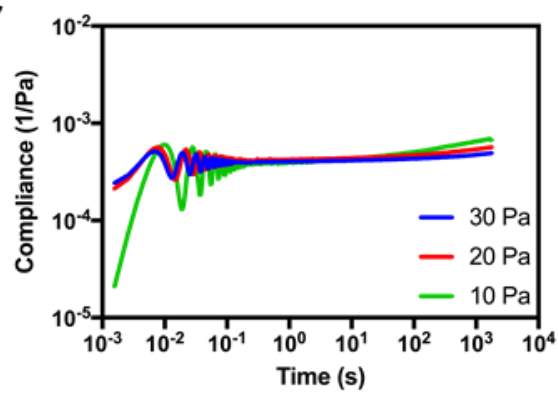

e

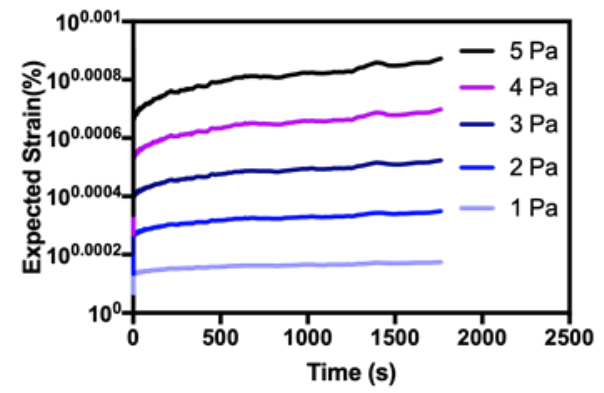

Fig. S2. Rheology assessment of DLSH formulations. (a) Tan $(\delta)$ versus stress test showed that all formulations presented a viscoelastic behavior $[\tan (\delta)<1]$ under 0.1 to $1000 \mathrm{~Pa}$ stress. Compliance versus time curves using 10, 20, and $30 \mathrm{~Pa}$ were recorded in the S (b) and SP (c) compositions confirming their viscoelastic behavior under stress. Creep response of $S$ (d) and SP (e) formulations was measured and calculated by using expected physiological strain levels. 
Figure S3

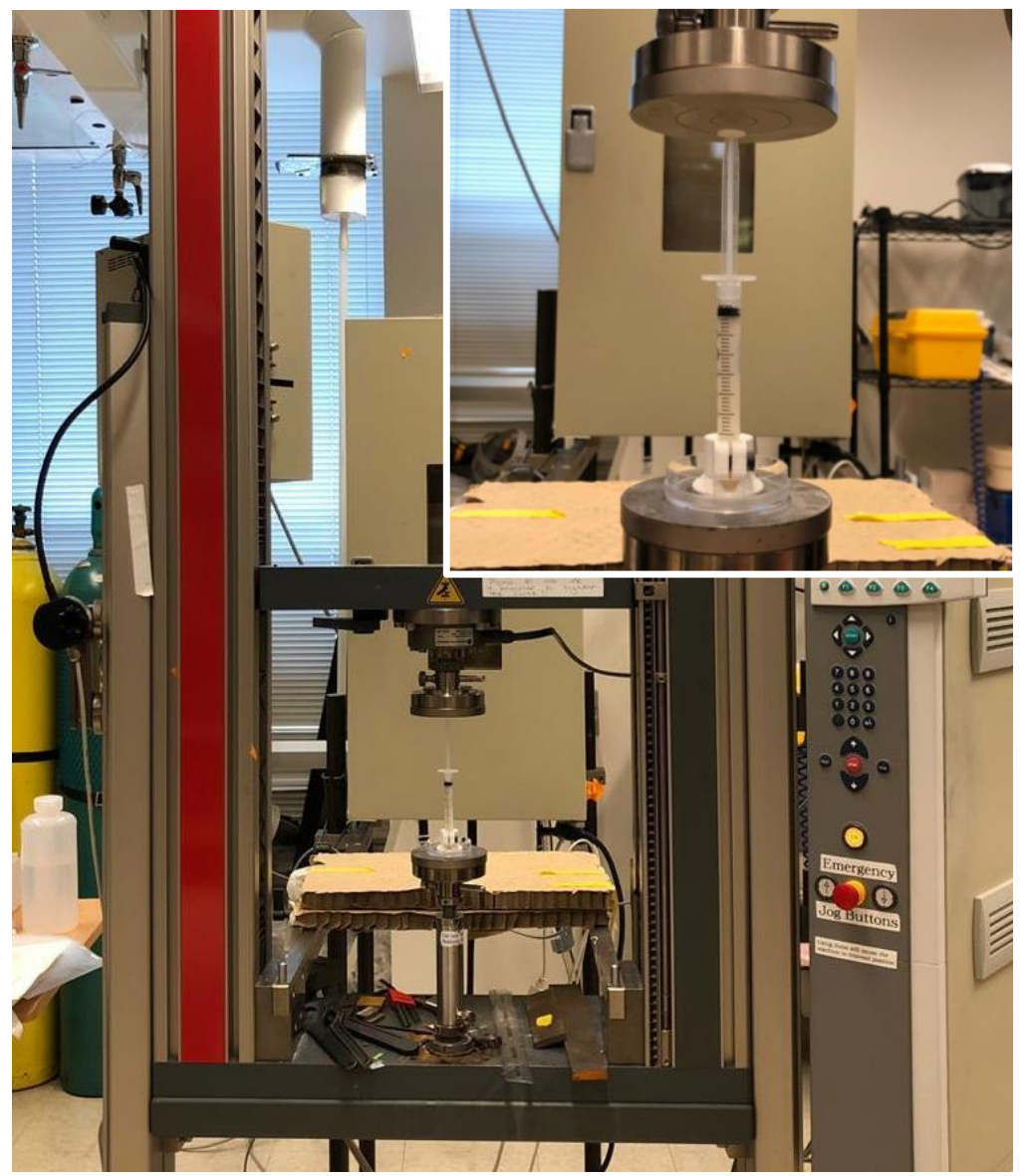

Fig. S3. Mechanical Tester Setup. A mechanical tester instrument was used to determine hydrogel extrusion force. 
Figure S4

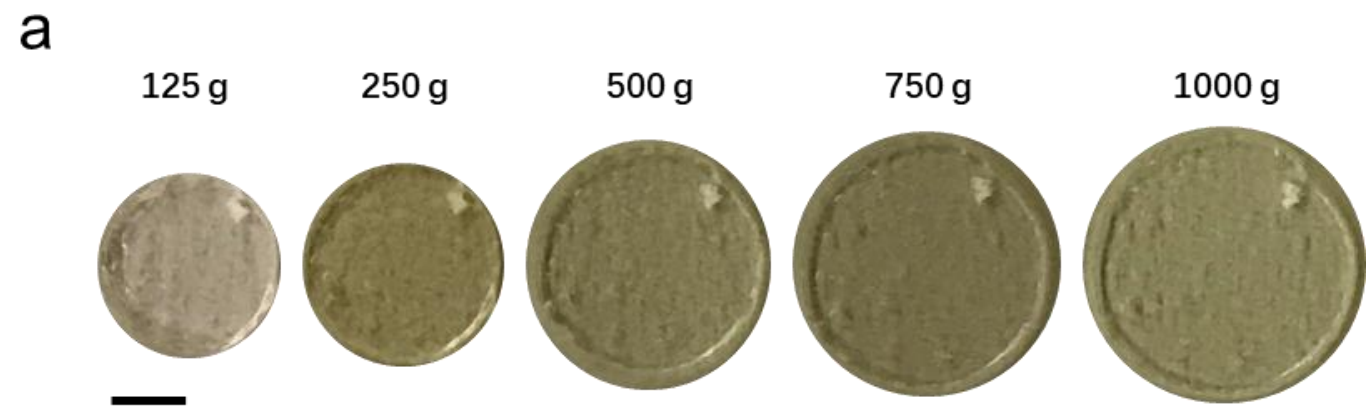

b

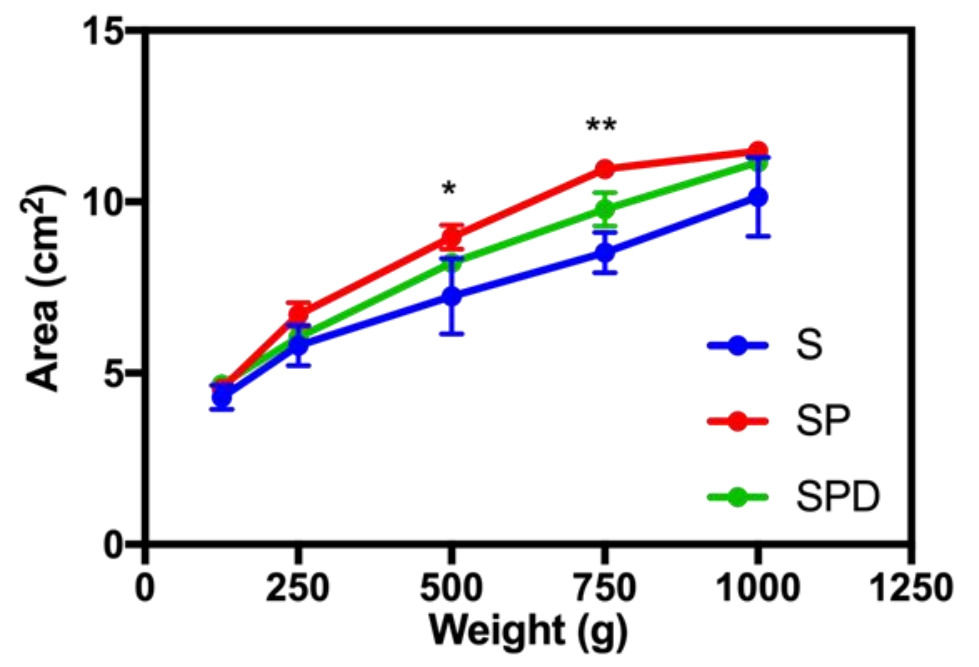

Fig. S4. Spread area of SPD formulation. (a) Representative photographs of SPD formulation after compression with $125 \mathrm{~g}, 250 \mathrm{~g}, 500 \mathrm{~g}, 750 \mathrm{~g}$, and $1000 \mathrm{~g}$. (b) Spread area of hydrogel samples was measured at room temperature. Scale bar $=1 \mathrm{~cm}$. 
Figure S5
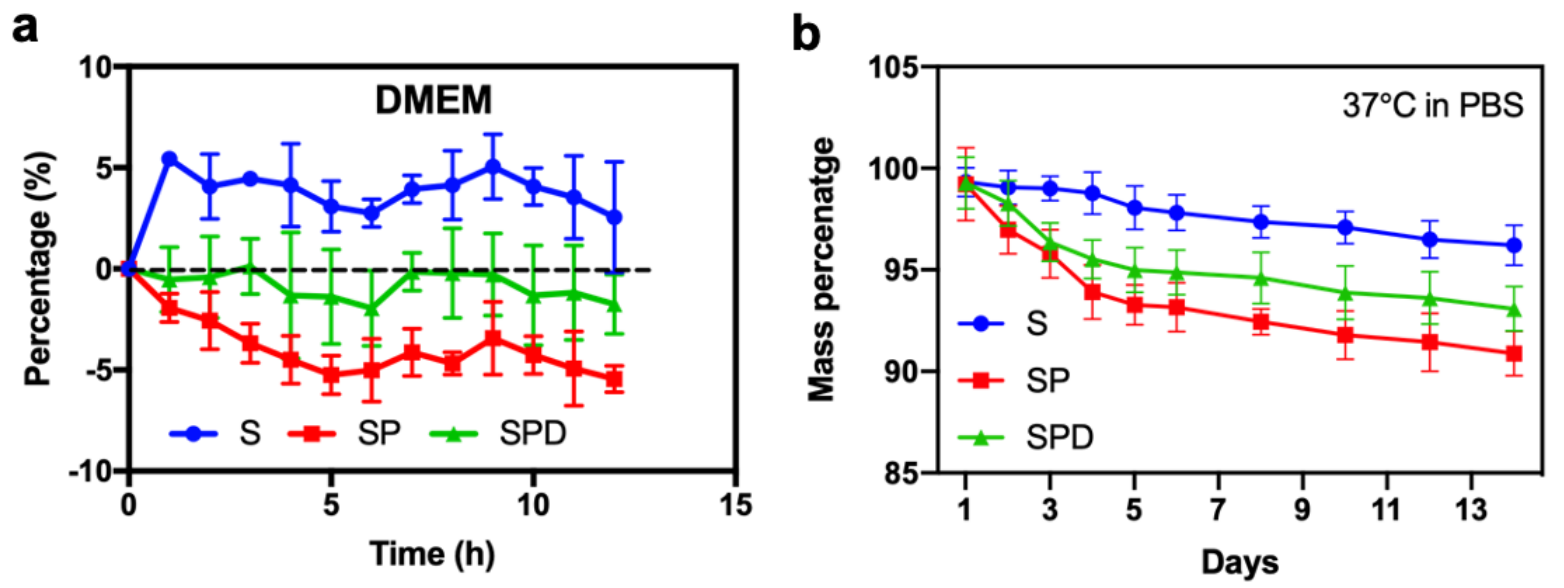

Fig. S5. (a) Hydrogel swelling was determined by changes on weight percentage after incubation in Dulbecco's Modified Eagle Medium (DMEM) at $37^{\circ} \mathrm{C}$. (b) Hydrogel degradation kinetics was determined by changes on weight percentage after incubation in Phosphate Buffered Saline (PBS) at $37^{\circ} \mathrm{C}$ in a period of 14 days. 


\section{Figure S6}

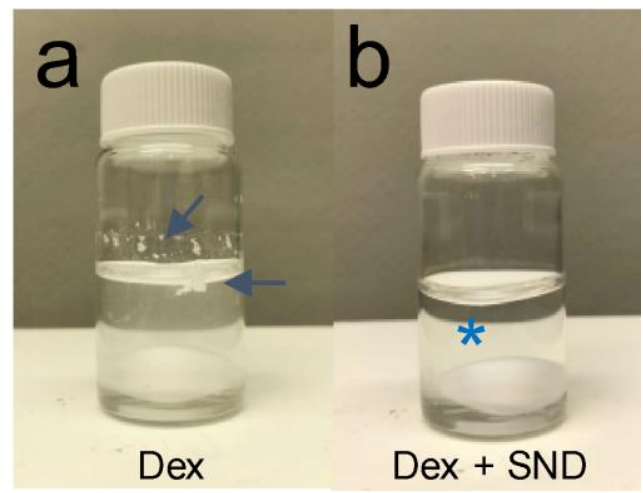

Fig. S6. DEX solubilization. (a) DEX solubilization in a water solution was not possible to achieve after 12 hours of continuous stirring at room temperature. (b) In contrast, solubilization of DEX in a SND solution was achieved at room temperature after 12 hours of continuous stirring. Arrows indicate the presence of DEX aggregates floating, and the asterisk indicates a transparent aqueous solution without drug clusters. 
Figure $\mathbf{S 7}$
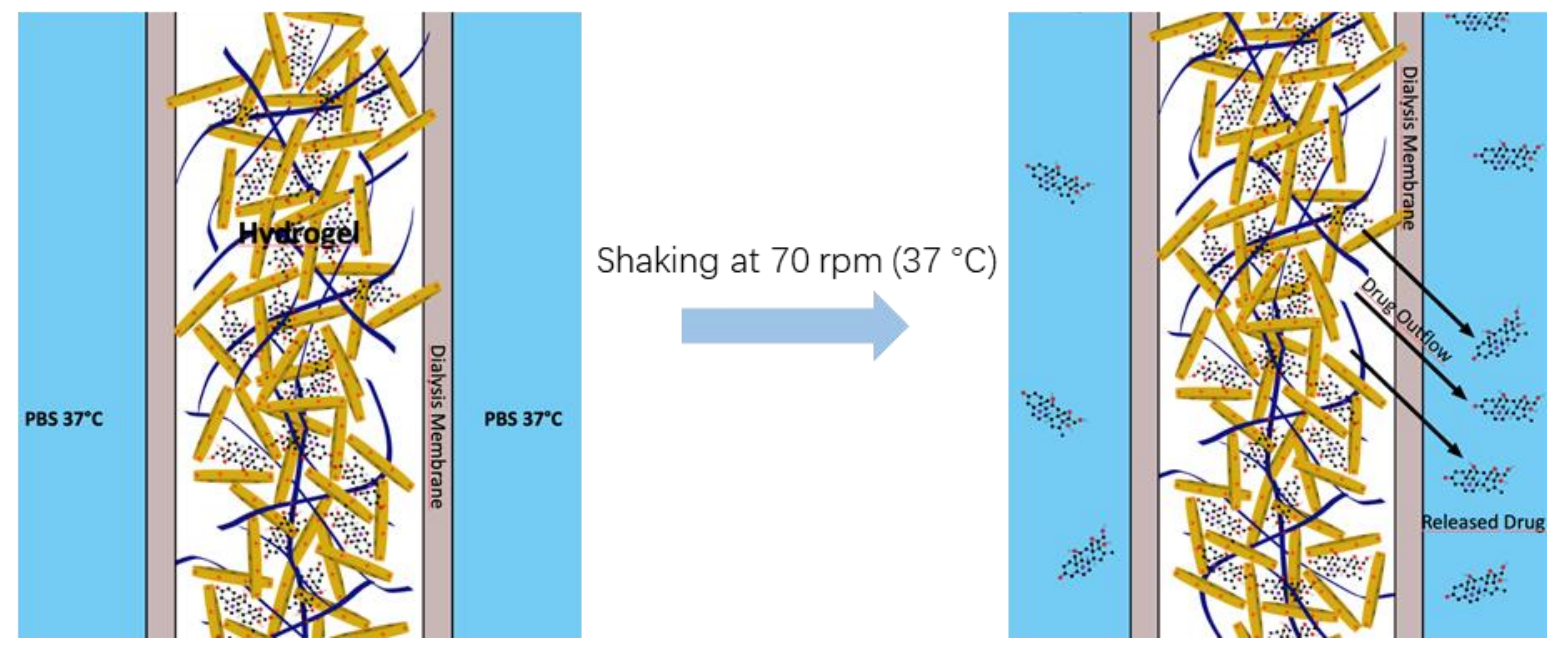

Fig. S7. Schematic representation of the set up used to determine DEX release

kinetics. A dialysis tube containing the DEX loaded hydrogel was incubated on PBS $(\mathrm{pH}$ 7.4) at $37^{\circ} \mathrm{C}$ under continuous shaking (70 rpm). 


\section{Figure S8}

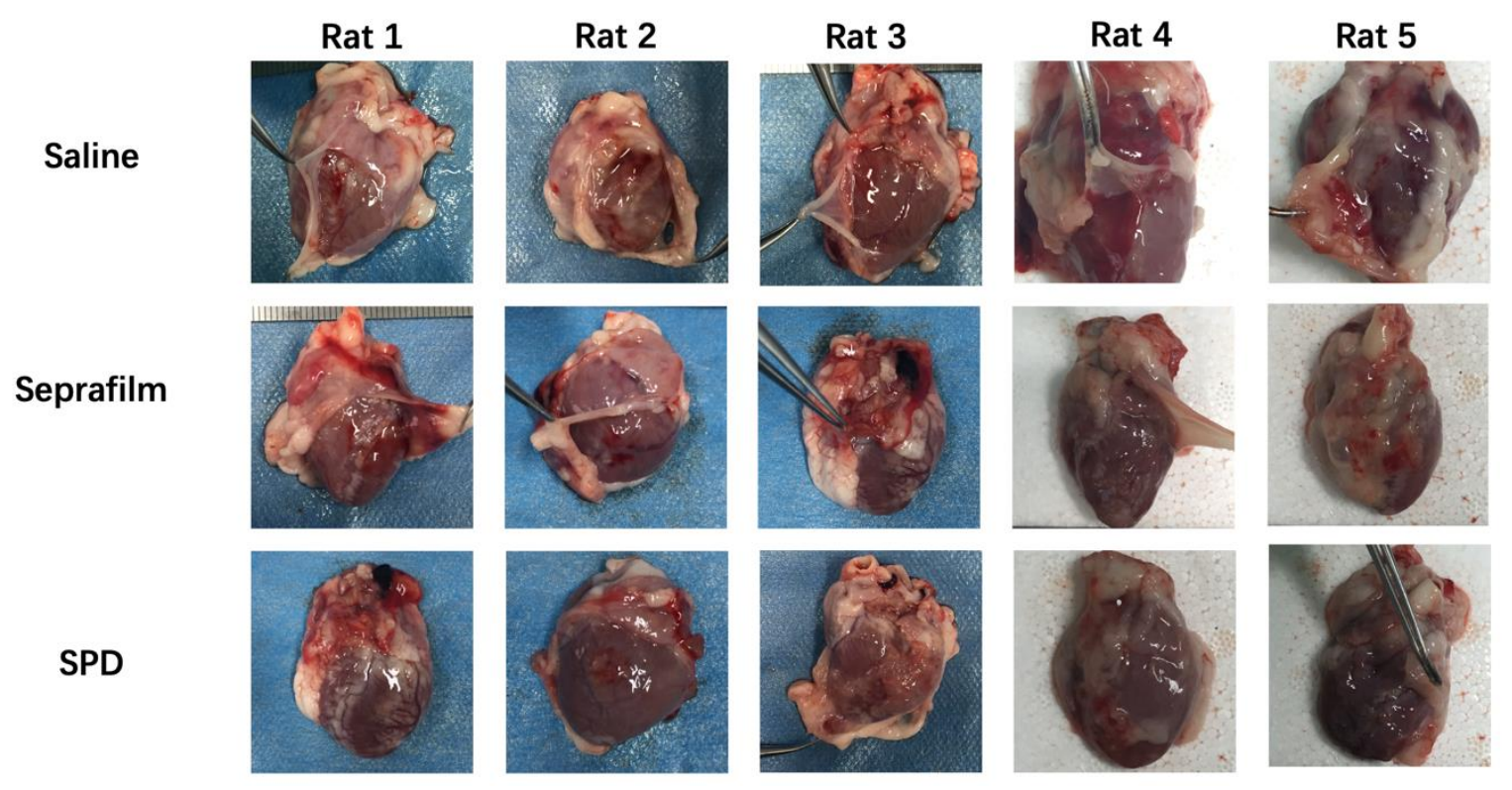

Fig. S8. Representative images of the gross appearance of the cardiac samples in each group (Saline, Seprafilm and SPD hydrogel), harvested 7 days after application. 
Figure S9

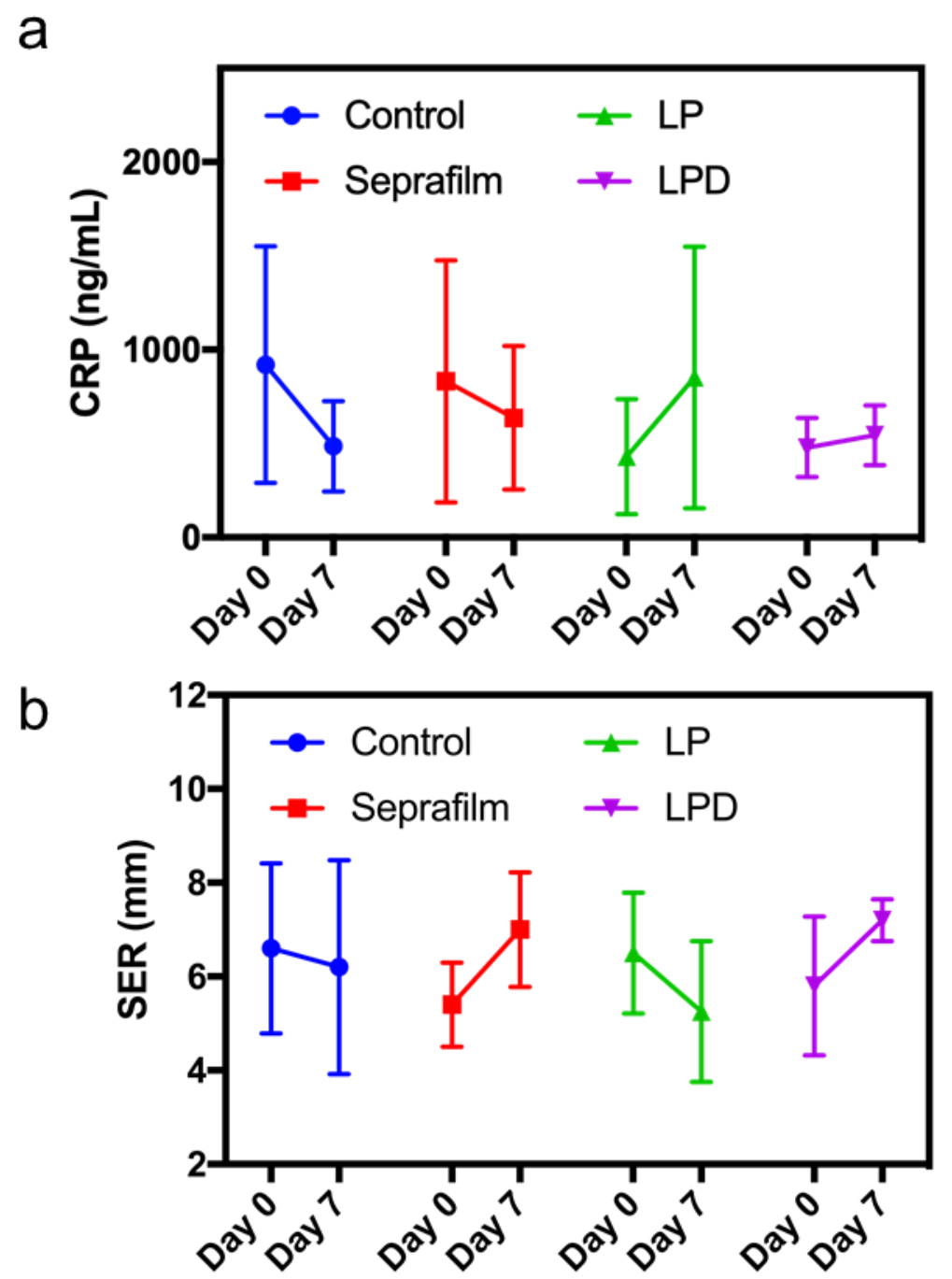

Fig. S9. Determination of systemic inflammation. (a) C-reactive protein (CRP) and (b) erythrocyte sedimentation rate (ESR) tests showed no significant systemic inflammatory response between pre-surgical (Day 0) and post-surgical animals (Day 7) across the different groups. 
Figure S10

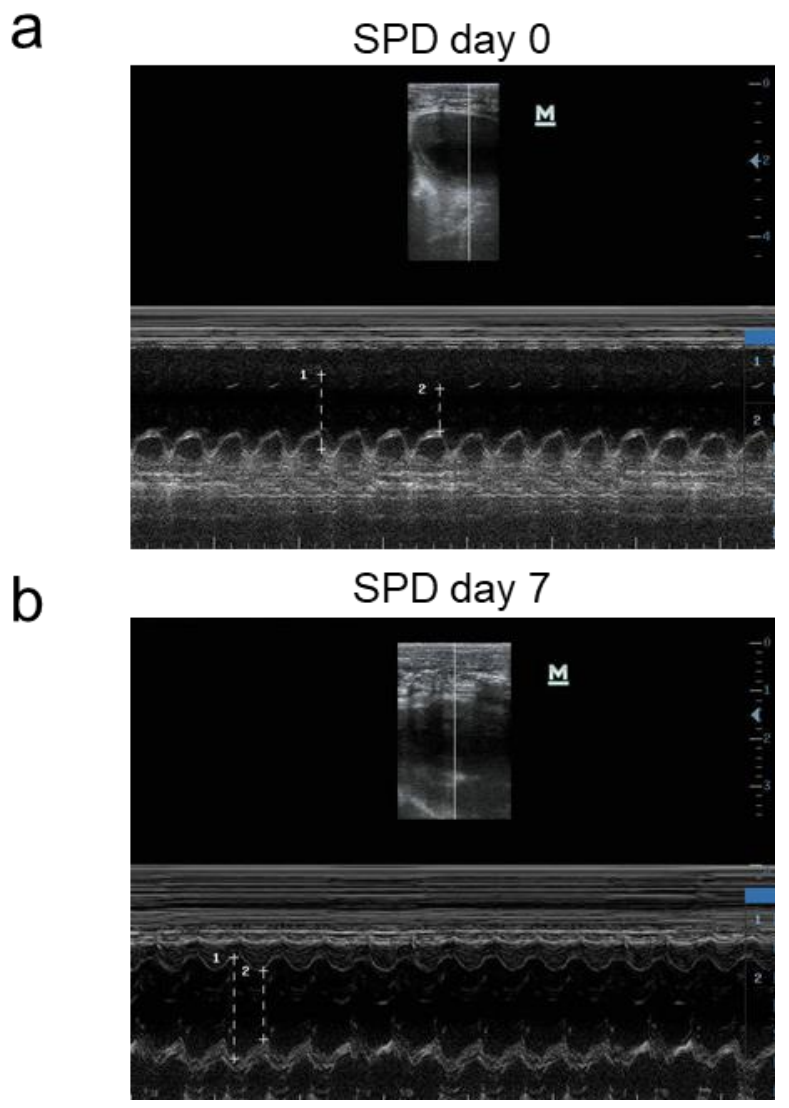

Fig. S10. Echocardiographic assessment of the heart. (a) Representative image of an echocardiogram showing intact pericardial and epicardial layers before surgery. (b) Echocardiographic image of an animal from the SPD group showing an intact intrapericardial space; no fibrotic adhesions can be observed after 7 postoperative days. 
Table S1

\begin{tabular}{|c|c|c|c|}
\hline Needle & $\begin{array}{c}\text { Intraluminal } \\
\text { diameter }(\mathrm{mm})\end{array}$ & $\begin{array}{c}\text { Needle Length } \\
(\mathrm{mm})\end{array}$ & $\begin{array}{c}\text { Extrusion speed } \\
(\mathrm{mL} / \mathrm{min})\end{array}$ \\
\hline $18 G$ & $0.838 \pm 0.038$ & 25 & 2.0 \\
\hline $23 G$ & $0.337 \pm 0.019$ & 25 & 2.0 \\
\hline $26 G$ & $0.260 \pm 0.019$ & 13 & 2.0 \\
\hline
\end{tabular}

Table S1. Needle characteristics and extrusion speed. 
Table S2

\begin{tabular}{|c|c|c|}
\hline Types of hydrogel & Diameter $\varnothing(\mathrm{mm})$ & Formulations \\
\hline Fluid Gel & $\varnothing>24$ & No formulation \\
\hline Semi-fluid Gel & $19<\varnothing<24$ & No formulation \\
\hline Semi-stiff Gel & $16<\varnothing<19$ & No formulation \\
\hline Stiff Gel & $14<\varnothing<16$ & No formulation \\
\hline Very Stiff Gel & $\varnothing<14$ & S, SP, SPD \\
\hline
\end{tabular}

Table S2. Categorization of hydrogel stiffness. 
Table S3

\begin{tabular}{|c|c|c|c|}
\hline $\begin{array}{c}\text { Components present } \\
\text { in the SPD spectrum }\end{array}$ & Proton(s) & $\begin{array}{c}\text { Chemical shift }(\boldsymbol{\delta}) \\
(\mathbf{p m})\end{array}$ & Multiplicity \\
\hline PEO & bH & $3.84-3.43$ & $\mathrm{~m}$ \\
\hline DEX & $\mathrm{AH}$ & $7.64-7.44$ & $\mathrm{br} \mathrm{s}$ \\
\hline DEX & $\mathrm{BH}$ & $6.49-6.33$ & $\mathrm{br} \mathrm{s}$ \\
\hline DEX & $\mathrm{CH}$ & $6.27-6.14$ & $\mathrm{br} \mathrm{s}$ \\
\hline DEX & $\mathrm{DCH}_{3}$ & 1.72 & $\mathrm{~s}$ \\
\hline DEX & $\mathrm{ECH}_{3}$ & 1.15 & $\mathrm{~s}$ \\
\hline DEX & $\mathrm{FCH}_{3}$ & 1.05 & $\mathrm{~s}$ \\
\hline
\end{tabular}

Table S3. Chemical shift peak of each Proton corresponding to components in the SPD hydrogel. 
Table S4

\begin{tabular}{|c|c|l|}
\hline Adhesion region & Adhesion grade & Adhesion evaluation: \\
\hline Injury site 1 & 0 & No adhesions \\
\hline Injury site 2 & 1 & Filmy adhesions, blunt dissection \\
\hline Injury site 3 & 2 & \begin{tabular}{l} 
Strong adhesions, sharp dissection \\
\hline Injury site 4
\end{tabular} \\
\hline
\end{tabular}

Table S4. Modified peritoneal adhesion index (PAI) adapted for intrapericardial adhesion grading. 
Supplemental Movie 1. Echocardiographic analysis of the heart of a control animal after 7 postoperative days. Pathological thickening of the pericardium and an echolucent structure in the intrapericardial space were observed and indicate the presence of fibrotic adhesions. 
Supplemental Movie 2. Echocardiographic analysis of the heart of an animal treated with the DLSH. A slight SPD deformation was observed, nevertheless, the hydrogel system was able to recover its mechanical integrity and remain protecting the epicardial surface. 